\title{
Global behavior of a third-order rational difference equation
}

\author{
Lama Sh. Aljoufi ${ }^{\mathrm{a}}$, A. M. Ahmed ${ }^{\mathrm{a}, \mathrm{b}, *}$, Samir Al Mohammady ${ }^{\mathrm{a}, \mathrm{c}}$ \\ ${ }^{a}$ Department of Mathematics, College of Science, Jouf University, P.O. Box 2014, Sakaka, Jouf, Saudi Arabia. \\ ${ }^{b}$ Department of Mathematics, Faculty of Science, Al Azhar University, Nasr City 11884, Cairo, Egypt. \\ ${ }^{c}$ Department of Mathematics, Faculty of Science, Helwan University, Helwan 11795, Egypt.
}

\begin{abstract}
In this paper, we investigate the behavior of solutions of the difference equation

$$
x_{n+1}=\frac{\alpha\left(x_{n-1}+x_{n-2}\right)+(\alpha-1) x_{n-1} x_{n-2}}{x_{n-1} x_{n-2}+\alpha}, \quad n=0,1,2, \ldots
$$

where the initial conditions $x_{-2}, x_{-1}, x_{0}$ are arbitrary non-negative real numbers and the parameter $\alpha \in[1, \infty)$. More precisely, we study the boundedness, stability, and oscillation of the solutions of this equation.
\end{abstract}

Keywords: Difference equations, stability, oscillation.

2020 MSC: 39A10, 39A21, 39A30.

(C)2022 All rights reserved.

\section{Introduction}

The theory of difference equations is a very rich research field. It was appeared already at the beginning of the last century in intensive works by the authors. A few years ago, the classes of difference equations attracted much attention. They have been the object of some intensive studies by many authors. The theory of these types of equations was one of the most important concepts in mathematics and appears naturally in numerous scientific problems that have been widely applied in discretization methods for differential equations, population biology, medicine, economic, physics, probability theory, genetics, and psychology. Very recently, the study of nonlinear difference equations has been a lot of interest in studying the global attractivity, boundedness character, periodicity and the solution form. For example, Abu-Saris et al [1] investigated the asymptotic stability of the difference equation

$$
x_{n+1}=\frac{a+x_{n} x_{n-k}}{x_{n}+x_{n-k}}, \quad n=0,1,2, \ldots,
$$

\footnotetext{
*Corresponding author

Email addresses: lamashuja11@gmail.com (Lama Sh. Aljoufi), ahmedelkb@yahoo.com \& amaahmed@ju.edu.sa (A. M. Ahmed), senssar@ju.edu.sa \& drsamirhnk@gmail.com (Samir Al Mohammady)

doi: $10.22436 /$ jmcs.025.03.08
}

Received: 2021-04-01 Revised: 2021-05-23 Accepted: 2021-06-16 
where $k$ is a nonnegative integer, $a \geqslant 0$ and $x_{-k}, \ldots, x_{0}>0$. Ahmed [3] investigated the behavior of solutions of the difference equation

$$
x_{n+1}=\frac{a+x_{n-1} x_{n-k}}{x_{n-1}+x_{n-k}}, \quad n=0,1,2, \ldots,
$$

where $k \in\{1,2\}, a \geqslant 0$ and $x_{-j}>0, j=0,1, \ldots, k$. Ma [19] investigated the global asymptotic stability for the higher-order difference equation

$$
z_{\mathrm{n}+1}=\frac{\mathrm{c}\left(z_{\mathrm{n}}+z_{\mathrm{n}-\mathrm{k}}\right)+(\mathrm{c}-1) z_{\mathrm{n}} z_{\mathrm{n}-\mathrm{k}}}{z_{\mathrm{n}} z_{\mathrm{n}-\mathrm{k}}+\mathrm{c}}, \mathrm{n}=0,1,2, \ldots,
$$

with positive initial values $z_{-k}, z_{-k+1}, \ldots, z_{0}$ and $c \in[1, \infty)$. For other related results, see $[2,4-18]$.

In this paper, we investigate the behavior of solutions of the difference equation

$$
x_{n+1}=\frac{\alpha\left(x_{n-1}+x_{n-2}\right)+(\alpha-1) x_{n-1} x_{n-2}}{x_{n-1} x_{n-2}+\alpha}, \quad n=0,1,2, \ldots,
$$

where the initial conditions $x_{-2}, x_{-1}, x_{0}$ are arbitrary non-negative real numbers and the parameter $\alpha \in$ $[1, \infty)$.

We need the following definitions.

Definition 1.1. Let I be an interval of real numbers and let

$$
f: \mathrm{I}^{\mathrm{k}+1} \rightarrow \mathrm{I}
$$

be a continuously differentiable function. Consider the difference equation

$$
x_{n+1}=f\left(x_{n}, x_{n-1}, \ldots, x_{n-k}\right), n=0,1, \ldots,
$$

with $x_{-k}, x_{-k+1}, \ldots, x_{0} \in I$. Let $\bar{x}$ be the equilibrium point of (1.1). The linearized equation of (1.1) about the equilibrium point $\bar{x}$ is

$$
y_{n+1}=c_{1} y_{n}+c_{2} y_{n-1}+\cdots+c_{k+1} y_{n-k},
$$

where $c_{1}=\frac{\partial f}{\partial x_{n}}(\bar{x}, \bar{x}, \ldots, \bar{x}), c_{2}=\frac{\partial f}{\partial x_{n-1}}(\bar{x}, \bar{x}, \ldots, \bar{x}), \ldots, c_{k+1}=\frac{\partial f}{\partial x_{n-k}}(\bar{x}, \bar{x}, \ldots, \bar{x})$. The characteristic equation of $(1.2)$ is

$$
\lambda^{k+1}-\sum_{i=1}^{k+1} c_{i} \lambda^{k-i+1}=0 .
$$

Definition 1.2. Let $\bar{x}$ be the equilibrium point of (1.1).

(i) The equilibrium point $\bar{x}$ of (1.1) is called locally stable if for every $\epsilon>0$, there exists $\delta>0$ such that for all $x_{-k}, x_{-k+1}, \ldots, x_{-1}, x_{0} \in I$ with

$$
\left|x_{-k}-\bar{x}\right|+\left|x_{-k+1}-\bar{x}\right|+\cdots+\left|x_{0}-\bar{x}\right|<\delta,
$$

we have

$$
\left|x_{n}-\bar{x}\right|<\epsilon \text { for all } n \geqslant-k \text {. }
$$

(ii) The equilibrium point $\bar{x}$ of (1.1) is called locally asymptotically stable if $\bar{x}$ is locally stable and there exists $\gamma>0$, such that for all $x_{-k}, x_{-k+1}, \ldots, x_{-1}, x_{0} \in I$ with

$$
\left|x_{-k}-\bar{x}\right|+\left|x_{-k+1}-\bar{x}\right|+\cdots+\left|x_{0}-\bar{x}\right|<\gamma,
$$

we have

$$
\lim _{n \rightarrow \infty} x_{n}=\bar{x} .
$$

(iii) The equilibrium point $\bar{x}$ of (1.1) is called global attractor if for all $x_{-k}, x_{-k+1}, \ldots, x_{-1}, x_{0} \in I$, we have

$$
\lim _{n \rightarrow \infty} x_{n}=\bar{x} .
$$


(iv) The equilibrium point $\bar{x}$ of (1.1) is called globally asymptotically stable if $\bar{x}$ is locally stable, and $\bar{x}$ is also a global attractor of (1.1).

(v) The equilibrium point $\bar{x}$ of (1.1) is called unstable if $\bar{x}$ is not locally stable.

Definition 1.3. A positive semicycle of $\left\{x_{n}\right\}_{n=-k}^{\infty}$ of (1.1) consists of a 'string' of terms $\left\{x_{l}, x_{l+1}, \ldots, x_{m}\right\}$, all greater than or equal to $\bar{x}$, with $l \geqslant-k$ and $m \leqslant \infty$ and such that either $l=-k$ or $l>-k$ and $x_{l-1}<\bar{x}$ and either $m=\infty$ or $m<\infty$ and $x_{m+1}<\bar{x}$.

A negative semicycle of $\left\{x_{n}\right\}_{\mathfrak{n}=-k}^{\infty}$ of (1.1) consists of a 'string' of terms $\left\{x_{l}, x_{l+1}, \ldots, x_{m}\right\}$, all less than $\bar{x}$, with $l \geqslant-k$ and $m \leqslant \infty$ and such that either $l=-k$ or $l>-k$ and $x_{l-1} \geqslant \bar{x}$ and either $m=\infty$ or $m<\infty$ and $x_{m+1} \geqslant \bar{x}$.

Definition 1.4. A solution $\left\{x_{n}\right\}_{n=-k}^{\infty}$ of (1.1) is called nonoscillatory if there exists $N \geqslant-k$ such that either

$$
x_{\mathrm{n}} \geqslant \overline{\mathrm{x}} \forall \mathrm{n} \geqslant \mathrm{N} \text { or } x_{\mathrm{n}}<\overline{\mathrm{x}} \forall \mathrm{n} \geqslant \mathrm{N},
$$

and it is called oscillatory if it is not nonoscillatory.

We need the following theorem.

Theorem 1.5 ([18]).

(i) If all roots of (1.3) have absolute value less than one, then the equilibrium point $\bar{x}$ of (1.1) is locally asymptotically stable.

(ii) If at least one of the roots of (1.3) has absolute value greater than one, then the equilibrium point $\bar{x}$ of (1.1) is unstable.

\section{Main results}

In this section, we investigate the behavior of solutions of equation.

$$
x_{n+1}=\frac{\alpha\left(x_{n-1}+x_{n-2}\right)+(\alpha-1) x_{n-1} x_{n-2}}{x_{n-1} x_{n-2}+\alpha}, \quad n=0,1,2, \ldots,
$$

where the initial conditions $x_{-2}, x_{-1}, x_{0}$ are arbitrary non-negative real numbers and the parameter $\alpha \in$ $[1, \infty)$. More precisely, we study the boundedness, stability, and oscillation of the solutions of (2.1).

Theorem 2.1. (2.1) has two equilibrium points $\bar{x}_{1}=0$ and $\bar{x}_{2}=\alpha$.

Proof. From (2.1), we can write

then we have

$$
\bar{x}=\frac{2 \alpha \bar{x}+(\alpha-1) \bar{x}^{2}}{\bar{x}^{2}+\alpha}
$$

$$
\bar{x}_{1}=0 \text { and } \bar{x}_{2}=\alpha .
$$

Theorem 2.2. The equilibrium point $\bar{x}_{1}=0$ of (2.1) is unstable equilibrium point.

Proof. The linearized equation of (2.1) about the equilibrium point $\bar{x}_{1}=0$ is

$$
y_{n+1}=y_{n-1}+y_{n-2},
$$

and so the characteristic equation of (2.2) about the equilibrium point $\bar{x}_{1}=0$ is

$$
\lambda^{3}-\lambda-1=0
$$

Suppose that

$$
f(\lambda)=\lambda^{3}-\lambda-1
$$

It is clear that $f(\lambda)$ has a root in the interval $(1, \infty)$ and so $\bar{x}_{1}=0$ is an unstable equilibrium point. This completes the proof. 
Theorem 2.3. The equilibrium point $\bar{x}_{2}=\alpha$ of (2.1) is locally asymptotically stable.

Proof. The linearized equation of (2.1) about the equilibrium point $\bar{x}_{2}=\alpha$ is

$$
y_{n+1}=0,
$$

and so the characteristic equation of (2.3) about the equilibrium point $\bar{x}_{2}=\alpha$ is

$$
\lambda^{3}=0,
$$

which implies that $\left|\lambda_{1}\right|=\left|\lambda_{2}\right|=\left|\lambda_{3}\right|=0<1$, from which the proof is completed.

Lemma 2.4. The following identities are true

(i)

(ii)

$$
x_{n+1}-\alpha=\frac{\left(\alpha-x_{n-2}\right)\left(x_{n-1}-\alpha\right)}{x_{n-1} x_{n-2}+\alpha}, \text { for } n \geqslant 0
$$

$$
x_{n+1}-x_{n-1}=\frac{x_{n-2}\left(x_{n-1}+1\right)\left(\alpha-x_{n-1}\right)}{x_{n-1} x_{n-2}+\alpha}, \text { for } n \geqslant 0
$$

(iii)

(iv)

$$
x_{n+1}-x_{n-2}=\frac{x_{n-1}\left(x_{n-2}+1\right)\left(\alpha-x_{n-2}\right)}{x_{n-1} x_{n-2}+\alpha}, \text { for } n \geqslant 0
$$

$$
x_{n+1}-x_{n-3}=\frac{\left(\alpha-x_{n-3}\right)\left(1+x_{n-3}\right)\left(x_{n-2} x_{n-4}(\alpha-1)+\alpha\left(x_{n-2}+x_{n-4}\right)\right)}{\left(x_{n-1} x_{n-2}+\alpha\right)\left(x_{n-3} x_{n-4}+\alpha\right)}, \text { for } n \geqslant 2
$$

(v)

$$
x_{n+1}-x_{n-4}=\frac{\left(\alpha-x_{n-4}\right)\left(1+x_{n-4}\right)\left(x_{n-2} x_{n-3}(\alpha-1)+\alpha\left(x_{n-2}+x_{n-3}\right)\right)}{\left(x_{n-1} x_{n-2}+\alpha\right)\left(x_{n-3} x_{n-4}+\alpha\right)}, \text { for } n \geqslant 3
$$

Proof.

(i)

$$
x_{n+1}-\alpha=\frac{\alpha\left(x_{n-1}+x_{n-2}\right)+(\alpha-1) x_{n-1} x_{n-2}}{x_{n-1} x_{n-2}+\alpha}-\alpha=\frac{\left(\alpha-x_{n-2}\right)\left(x_{n-1}-\alpha\right)}{x_{n-1} x_{n-2}+\alpha}, \text { for } n \geqslant 0
$$

(ii)

$$
x_{n+1}-x_{n-1}=\frac{\alpha\left(x_{n-1}+x_{n-2}\right)+(\alpha-1) x_{n-1} x_{n-2}}{x_{n-1} x_{n-2}+\alpha}-x_{n-1}=\frac{x_{n-2}\left(x_{n-1}+1\right)\left(\alpha-x_{n-1}\right)}{x_{n-1} x_{n-2}+\alpha}, \text { for } n \geqslant 0 .
$$

(iii)

$$
x_{n+1}-x_{n-2}=\frac{\alpha\left(x_{n-1}+x_{n-2}\right)+(\alpha-1) x_{n-1} x_{n-2}}{x_{n-1} x_{n-2}+\alpha}-x_{n-2}=\frac{x_{n-1}\left(x_{n-2}+1\right)\left(\alpha-x_{n-2}\right)}{x_{n-1} x_{n-2}+\alpha}, \text { for } n \geqslant 0 .
$$

(iv)

$$
\begin{aligned}
x_{n+1}-x_{n-3} & =\frac{\alpha\left(x_{n-1}+x_{n-2}\right)+(\alpha-1) x_{n-1} x_{n-2}}{x_{n-1} x_{n-2}+\alpha}-x_{n-3} \\
& =\frac{\alpha\left(\frac{\alpha\left(x_{n-3}+x_{n-4}\right)+(\alpha-1) x_{n-3} x_{n-4}}{x_{n-3} x_{n-4}+\alpha}+x_{n-2}\right)+(\alpha-1)\left(\frac{\alpha\left(x_{n-3}+x_{n-4}\right)+(\alpha-1) x_{n-3} x_{n-4}}{x_{n-3} x_{n-4}+\alpha}\right) x_{n-2}}{x_{n-1} x_{n-2}+\alpha}-x_{n-3} \\
& =\frac{\left(\alpha-x_{n-3}\right)\left(1+x_{n-3}\right)\left(x_{n-2} x_{n-4}(\alpha-1)+\alpha\left(x_{n-2}+x_{n-4}\right)\right)}{\left(x_{n-1} x_{n-2}+\alpha\right)\left(x_{n-3} x_{n-4}+\alpha\right)}, \text { for } n \geqslant 2 .
\end{aligned}
$$


(v)

$$
\begin{aligned}
x_{n+1}-x_{n-4} & =\frac{\alpha\left(x_{n-1}+x_{n-2}\right)+(\alpha-1) x_{n-1} x_{n-2}}{x_{n-1} x_{n-2}+\alpha}-x_{n-4} \\
& =\frac{\alpha\left(\frac{\alpha\left(x_{n-3}+x_{n-4}\right)+(\alpha-1) x_{n-3} x_{n-4}}{x_{n-3} x_{n-4}+\alpha}+x_{n-2}\right)+(\alpha-1)\left(\frac{\alpha\left(x_{n-3}+x_{n-4}\right)+(\alpha-1) x_{n-3} x_{n-4}}{x_{n-3} x_{n-4}+\alpha}\right) x_{n-2}}{x_{n-1} x_{n-2}+\alpha}-x_{n-4} \\
& =\frac{\left(\alpha-x_{n-4}\right)\left(1+x_{n-4}\right)\left(x_{n-2} x_{n-3}(\alpha-1)+\alpha\left(x_{n-2}+x_{n-3}\right)\right)}{\left(x_{n-1} x_{n-2}+\alpha\right)\left(x_{n-3} x_{n-4}+\alpha\right)}, \text { for } n \geqslant 3
\end{aligned}
$$

Then, the proof is completed.

Theorem 2.5. If $\left\{x_{n}\right\}_{n=-2}^{\infty}$ be a solution to the rational nonlinear difference (2.1), then for $n \geqslant 0$ we have

(i) $x_{n+1}<x_{n-1}$, iff $x_{n-1}>\alpha$;

(ii) $x_{n+1}>x_{n-1}$, iff $x_{n-1}<\alpha$;

(iii) $x_{n+1}<x_{n-2}$, iff $x_{n-2}>\alpha$;

(iv) $x_{n+1}>x_{n-2}$, iff $x_{n-2}<\alpha$.

Proof. Let $x_{n-1}>\alpha\left(x_{n-1}<\alpha\right)$. Then from (2.5) we have $x_{n+1}<x_{n-1}\left(x_{n+1}>x_{n-1}\right)$. Also if $x_{n+1}<$ $x_{n-1}\left(x_{n+1}>x_{n-1}\right)$, then from (2.5) we have $x_{n-1}>\alpha\left(x_{n-1}<\alpha\right)$. Then the proofs of (i) and (ii) are completed.

Now, let $x_{n-2}>\alpha\left(x_{n-2}<\alpha\right)$. Then from (2.6) we have $x_{n+1}<x_{n-2}\left(x_{n+1}>x_{n-2}\right)$. Also if $x_{n+1}<$ $x_{n-2}\left(x_{n+1}>x_{n-2}\right)$, then from (2.6) we have $x_{n-2}>\alpha\left(x_{n-2}<\alpha\right)$. Then the proofs of (iii) and (iv) are completed.

Theorem 2.6. Let $\left\{x_{n}\right\}_{n=-2}^{\infty}$ be a solution of (2.1). Then the following statements are true.

(i) If $\mathrm{x}_{\mathrm{n}_{0}}=\overline{\mathrm{x}}_{2}=\alpha$, for some $\mathrm{n}_{0} \in\{-1,0,1,2, \ldots\}$, then $\mathrm{x}_{\mathrm{n}}=\overline{\mathrm{x}}_{2}=\alpha$, for all $\mathrm{n} \geqslant \mathrm{n}_{0}+2$. Also if $\mathrm{x}_{-2}=\overline{\mathrm{x}}_{2}=\alpha$, then $x_{n}=\bar{x}_{2}=\alpha$, for all $n \geqslant 3$.

(ii) If $x_{n_{0}}, x_{n_{0}+1}, x_{n_{0}+2}<\bar{x}_{2}=\alpha$, for some $n_{0} \in\{-2,-1,0,1,2, \ldots\}$, then $x_{n}<\bar{x}_{2}=\alpha$, for all $n \geqslant n_{0}$.

(iii) If (i) and (ii) are not satisfied, then $\left\{x_{n}\right\}_{n=-2}^{\infty}$ oscillates about $\bar{x}_{2}=\alpha$, with positive semicycles of length at most three, and negative semicycles of length at most two.

Proof.

(i) Let $x_{n_{0}}=\bar{x}_{2}=\alpha$, for some $n_{0} \in\{-1,0,1,2, \ldots\}$. Then from (2.4) we have $x_{n}=\bar{x}_{2}=\alpha$, for all $n \geqslant n_{0}+2$. If $x_{-2}=\bar{x}_{2}=\alpha$, then from (2.4) we have $x_{1}=\bar{x}_{2}=\alpha$, which implies that $x_{n}=\bar{x}_{2}=\alpha$, for all $n \geqslant 3$.

(ii) Let $x_{n_{0}}, x_{n_{0}+1}, x_{n_{0}+2}<\bar{x}_{2}=\alpha$, for some $n_{0} \in\{-2,-1,0,1,2, \ldots\}$. Then from (2.4) we have $x_{n}<\bar{x}_{2}=\alpha$, for all $n \geqslant n_{0}$.

(iii) Suppose without loss of generality that there exists $n_{0} \in\{-2,-1,0,1,2, \ldots\}$, such that $x_{n_{0}}, x_{n_{0}+1}, x_{n_{0}+2}>$ $\bar{x}_{2}=\alpha$. Then from (2.4) we have $x_{\mathfrak{n}_{0}+3}, x_{\mathfrak{n}_{0}+4}<\alpha, x_{\mathfrak{n}_{0}+5}>\alpha, x_{\mathfrak{n}_{0}+6}<\alpha$ and $x_{\mathfrak{n}_{0}+7}, x_{\mathfrak{n}_{0}+8}, x_{\mathfrak{n}_{0}+9}>\bar{x}_{2}=\alpha$. The proofs of the other possibilities are similar, and will be omitted.

Theorem 2.7. The equilibrium point $\overline{\mathrm{x}}_{2}=\alpha$ of (2.1) is globally asymptotically stable.

Proof. We know by Theorem 2.3 that the equilibrium point $\bar{x}_{2}=\alpha$ of (2.1) is locally asymptotically stable, and so it suffices to show that $\lim _{n \rightarrow \infty} x_{n}=\bar{x}_{2}=\alpha$. If there exists $n_{0} \in\{-2,-1,0,1,2, \ldots\}$, such that $x_{n_{0}}=\bar{x}_{2}=\alpha$, then from Theorem 2.6 we have $\lim _{n \rightarrow \infty} x_{n}=\bar{x}_{2}=\alpha$. Also, if $x_{-2}, x_{-1}, x_{0}<\bar{x}_{2}=\alpha$, then by Theorem 2.6 we have $x_{n}<\bar{x}_{2}=\alpha$, for all $n \geqslant-2$ and from (2.5), we have $x_{n+1}>x_{n-1}$, for $n \geqslant 0$. So the subsequences $\left\{x_{2 n}\right\}_{\mathfrak{n}=0}^{\infty}$ and $\left\{x_{2 n-1}\right\}_{\mathfrak{n}=0}^{\infty}$ are increasing and bounded, which implies that the even terms $\left\{x_{2 n}\right\}_{n=0}^{\infty}$ converge to a limit (say $M_{1}>0$ ) and the odd terms $\left\{x_{2 n-1}\right\}_{n=0}^{\infty}$ converge to a limit (say $M_{2}>0$ ). Then

$$
M_{1}=\frac{\alpha\left(M_{1}+M_{2}\right)+(\alpha-1) M_{1} M_{2}}{M_{1} M_{2}+\alpha} \text { and } M_{2}=\frac{\alpha\left(M_{1}+M_{2}\right)+(\alpha-1) M_{1} M_{2}}{M_{1} M_{2}+\alpha}
$$




$$
\Longrightarrow M_{1}=M_{2} \quad \text { and } \quad M_{1}\left[\left(M_{1}+1\right)\left(M_{1}-\alpha\right)\right]=0,
$$

which implies that either $M_{1}=M_{2}=-1$, or $M_{1}=M_{2}=0$, or $M_{1}=M_{2}=\alpha$. Since $M_{1}=M_{2}>0$, then $M_{1}=M_{2}=\alpha$.

Now, suppose that $x_{-2}, x_{-1}, x_{0}>\bar{x}_{2}=\alpha$, then from (2.4), (2.5), and (2.6) we have $\alpha<\cdots<x_{14}<x_{7}<$ $x_{0}$, and so the sequence $\left\{x_{7 n}\right\}_{\mathfrak{n}=0}^{\infty}$ is decreasing and bounded, which implies that the sequence $\left\{x_{7 n}\right\}_{\mathfrak{n}=0}^{\infty}$ converges to a limit (say $\mathrm{L}_{0}>0$ ).

For the sequence $\left\{x_{7 n+1}\right\}_{n=0}^{\infty}$, we have from (2.4) that $0<x_{1}<\bar{x}_{2}=\alpha$, and from (2.4), (2.6), and (2.7) we have $0<x_{1}<x_{8}<x_{15}<\cdots<\alpha$, and so the sequence $\left\{x_{7 n+1}\right\}_{n=0}^{\infty}$ is increasing and bounded, which implies that the sequence $\left\{x_{7 n+1}\right\}_{n=0}^{\infty}$ converges to a limit (say $L_{1}>0$ ).

Similarly from (2.4)-(2.8), we have the following results.

The sequence $\left\{x_{7 n+2}\right\}_{\mathfrak{n}=0}^{\infty}$ is increasing and bounded, and so converges to a limit (say $L_{2}>0$ ).

The sequence $\left\{x_{7 n+3}\right\}_{n=0}^{\infty}$ is decreasing and bounded, and so converges to a limit (say $L_{3}>0$ ).

The sequence $\left\{x_{7 n+4}\right\}_{n=0}^{\infty}$ is increasing and bounded, and so converges to a limit (say $L_{4}>0$ ).

The sequence $\left\{x_{7 n+5}\right\}_{\mathfrak{n}=0}^{\infty}$ is decreasing and bounded, and so converges to a limit (say $L_{5}>0$ ).

The sequence $\left\{x_{7 n+6}\right\}_{n=0}^{\infty}$ is decreasing and bounded, and so converges to a limit (say $L_{6}>0$ ).

So we have from (2.1) that

$$
\begin{array}{ll}
\mathrm{L}_{0}=\frac{\alpha\left(\mathrm{L}_{4}+\mathrm{L}_{5}\right)+(\alpha-1) \mathrm{L}_{4} \mathrm{~L}_{5}}{\mathrm{~L}_{4} \mathrm{~L}_{5}+\alpha}, & \mathrm{L}_{1}=\frac{\alpha\left(\mathrm{L}_{5}+\mathrm{L}_{6}\right)+(\alpha-1) \mathrm{L}_{5} \mathrm{~L}_{6}}{\mathrm{~L}_{5} \mathrm{~L}_{6}+\alpha}, \quad \mathrm{L}_{2}=\frac{\alpha\left(\mathrm{L}_{0}+\mathrm{L}_{6}\right)+(\alpha-1) \mathrm{L}_{0} \mathrm{~L}_{6}}{\mathrm{~L}_{0} \mathrm{~L}_{6}+\alpha}, \\
\mathrm{L}_{3}=\frac{\alpha\left(\mathrm{L}_{0}+\mathrm{L}_{1}\right)+(\alpha-1) \mathrm{L}_{0} \mathrm{~L}_{1}}{\mathrm{~L}_{0} \mathrm{~L}_{1}+\alpha}, & \mathrm{L}_{4}=\frac{\alpha\left(\mathrm{L}_{1}+\mathrm{L}_{2}\right)+(\alpha-1) \mathrm{L}_{1} \mathrm{~L}_{2}}{\mathrm{~L}_{1} \mathrm{~L}_{2}+\alpha},
\end{array}
$$

If $\alpha=1$, then the solution of this system is either $L_{i}=-1, i=0,1, \ldots, 6$, or $L_{i}=0, i=0,1, \ldots, 6$, or $\mathrm{L}_{\mathrm{i}}=\alpha, i=0,1, \ldots, 6$.

If $\alpha \in(1, \infty)$, then the solution of this system is either $L_{i}=-1, i=0,1, \ldots, 6$, or $L_{i}=0, i=0,1, \ldots, 6$, or $L_{i}=\alpha, i=0,1, \ldots, 6$, or $L_{i}=0, i=0,4,5$ and $L_{i}=-\frac{2 \alpha}{-1+\alpha}, i=1,2,3,6$, or $L_{i}=0, i=2,3,5$ and $L_{i}=-\frac{2 \alpha}{-1+\alpha}, i=0,1,4,6$, or $L_{i}=0, i=1,5,6$ and $L_{i}=-\frac{2 \alpha}{-1+\alpha}, i=0,2,3,4$, or $L_{i}=0, i=0,1,3$ and $\mathrm{L}_{i}=-\frac{2 \alpha}{-1+\alpha}, i=2,4,5,6$, or $\mathrm{L}_{i}=0, i=0,2,6$ and $\mathrm{L}_{i}=-\frac{2 \alpha}{-1+\alpha}, i=1,3,4,5$, or $\mathrm{L}_{i}=0, i=3,4,6$ and $\mathrm{L}_{i}=-\frac{2 \alpha}{-1+\alpha}, i=0,1,2,5$, or $\mathrm{L}_{i}=0, i=1,2,4, \mathrm{~L}_{i}=-\frac{2 \alpha}{-1+\alpha}, i=0,3,5$ and $\mathrm{L}_{i}=\frac{8 \alpha}{(1-\alpha)^{3}}-\frac{12 \alpha}{(1-\alpha)^{2}}+\frac{6 \alpha}{(1-\alpha)}-$ $\frac{16 \alpha^{2}}{(1-\alpha)^{3}}+\frac{12 \alpha^{2}}{(1-\alpha)^{2}}+\frac{8 \alpha^{3}}{(1-\alpha)^{3}}, i=6$. Since $L_{i}>0, i=0,1, \ldots, 6$, so the only possible solution of this system in both two cases is $L_{i}=\alpha, i=0,1, \ldots, 6$, and so we have $\lim _{n \rightarrow \infty} x_{n}=\bar{x}_{2}=\alpha$.

The proofs for the other cases $x_{-2}, x_{-1}>\bar{x}_{2}=\alpha, x_{0}<\bar{x}_{2}=\alpha$, or $x_{-2}, x_{-1}<\bar{x}_{2}=\alpha, x_{0}>\bar{x}_{2}=\alpha$, or $x_{-2}>\bar{x}_{2}=\alpha, x_{-1}, x_{0}<\bar{x}_{2}=\alpha$, or $x_{-2}<\bar{x}_{2}=\alpha, x_{-1}, x_{0}>\bar{x}_{2}=\alpha$, or $x_{-2}>\bar{x}_{2}=\alpha, x_{-1}<\bar{x}_{2}=\alpha$, $x_{0}>\bar{x}_{2}=\alpha$ or $x_{-2}<\bar{x}_{2}=\alpha, x_{-1}>\bar{x}_{2}=\alpha, x_{0}<\bar{x}_{2}=\alpha$ are embedded in the proof of the last case, and will be omitted. Therefore the proof is completed.

Remark 2.8. When $\alpha=1$, we obtain the results due to Ahmed [3].

\section{Acknowledgment}

The authors are grateful to the referees for numerous comments that improved the quality of the paper.

\section{References}

[1] R. Abu-Saris, C. Cinar, I. Yalçinkaya, On the asymptotic stability of $x_{n+1}=\frac{a+x_{n} x_{n-k}}{x_{n}+x_{n-k}}$, Comput. Math. Appl., 56 (2008), 1172-1175. 1 
[2] A. M. Ahmed, On the dynamics of a higher-order rational difference equation, Discrete Dyn. Nat. Soc., 2011 (2011), 8 pages. 1

[3] A. M. Ahmed, On the Dynamics Of $x_{n+1}=\frac{a+x_{n-1} x_{n-k}}{x_{n-1}+x_{n-k}}$, J. Appl. Math. Inform., 32 (2014), 599-607. 1, 2.8

[4] A. M. Ahmed, H. M. El-Owaidy, A. Hamza, A. M. Youssef, on the recursive sequence $x_{n+1}=\frac{a+b x_{n-1}}{A+B x_{n}^{k}}$, J. Appl. Math. Inform., 27 (2009), 275-289. 1

[5] A. M. Ahmed, H. M. Rezk, On the dynamics of the recursive sequence $x_{n+1}=\frac{x_{n-1}}{p+q x_{n}^{2}+x_{n-1}}$, J. Pure Appl. Math. Adv. Applic., 14 (2015), 41-56.

[6] A. M. Ahmed, A. M. Youssef, A solution form of a class of higher-order rational difference equations, J. Egyptian Math. Soc., 21 (2013), 248-253.

[7] C. Cinar, On the Positive Solutions of the Difference Equation $x_{n+1}=\frac{x_{n-1}}{1+x_{n} x_{n-1}}$, Appl. Math. Comput., 150 (2004), 21-24.

[8] C. Cinar, On the Difference Equation $x_{n+1}=\frac{x_{n-1}}{-1+x_{n} x_{n-1}}$, Appl. Math. Comput., 158 (2004), 813-816.

[9] C. Cinar, On the Positive Solutions of the Difference Equation $x_{n+1}=\frac{a x_{n-1}}{1+b x_{n} x_{n-1}}$, Appl. Math. Comput., 156 (2004), 587-590.

[10] C. Cinar, R. Karatas, I. Yalçinkaya, On Solutions of the Difference Equation $x_{n+1}=\frac{x_{n-3}}{-1+x_{n} x_{n-1} x_{n-2} x_{n-3}}$, Math. Bohem., 132 (2007), 257-261.

[11] E. M. Elabbasy, H. El-Metwally, E. M. Elsayed, On the Difference Equations $x_{n+1}=\frac{\alpha x_{n-k}}{\beta+\gamma \prod_{i=0}^{k} x_{n-i}}$, J. Conc. Appl. Math., 5 (2007), 101-113.

[12] E. M. Elabbasy, H. El-Metwally, E. M. Elsayed, Qualitative Behavior of Higher Order Difference Equation, Soochow J. Math., 33 (2007), 861-873.

[13] E. M. Elabbasy, H. El-Metwally, E. M. Elsayed, On the Difference Equation $x_{n+1}=\frac{a_{0} x_{n}+a_{1} x_{n-1}+\ldots+a_{k} x_{n-k}}{b_{0} x_{n}+b_{1} x_{n}-1+\ldots+b_{k} x_{n}-k}$, Math. Bohem, 133 (2008), 133-147.

[14] H. El-Owaidy, A. Ahmed, A. Youssef, On the Dynamics of $x_{n+1}=\frac{b x_{n-1}^{2}}{A+B x_{n-2}}$, Rostock. Math. Kolloq., 59 (2005),

[15] H. El-Owaidy, A. Ahmed, A. Youssef, The Dynamics of the Recursive Sequence $x_{n+1}=\frac{\alpha x_{n-1}}{\beta+\gamma x_{n-2}^{p}}$, Appl. Math. Lett., 18 (2005), 1013-1018.

[16] E. M. Elsayed, Dynamics of a rational recursive sequence, Int. J. Difference Equ., 4 (2009), 185-200.

[17] E. M. Elsayed, Behavior of a Rational Recursive Sequences, Stud. Univ. Babeş-Bolyai Math., 56 (2011), $27-42$.

[18] V. L. Kocić, G. Ladas, Global behavior of nonlinear difference equations of higher order with applications, Kluwer Academic Publishers, Dordrecht, (1993). 1, 1.5

[19] W.-X. Ma, Global Behavior of a New Rational Nonlinear Higher-Order Difference Equation, Complexity, 2019 (2019), 4 pages. 1 